To appear in The Astronomical Journal, Vol. 124, July 2002

\title{
THE LUMINOSITY FUNCTIONS OF OLD AND INTERMEDIATE-AGE GLOBULAR CLUSTERS IN NGC 3610
}

\author{
BRADLEY C. WHITMORE \\ Space Telescope Science Institute, 3700 San Martin Drive, Baltimore, MD 21218
}

Electronic mail: whitmore@stsci.edu

FRANÇOIS SCHWEIZER, Carnegie Observatories, 813 Santa Barbara St., Pasadena, CA 91101-1292

Electronic mail: schweizer@ociw.edu

\author{
ARUNAV KUNDU, \\ Michigan State University, Physics \& Astronomy Department, East Lansing, MI 48824-1116 \\ Electronic mail: akundu@pa.msu.edu \\ and BRYAN W. MILLER \\ AURA/Gemini Observatory, Casilla 603, La Serena, Chile \\ Electronic mail: bmiller@gemini.edu
}

Received:

${ }^{1}$ Based on observations with the NASA/ESA Hubble Space Telescope, obtained at the Space Telescope Science Institute, which is operated by the Association of Universities for Research in Astronomy, Inc., under NASA contract NAS5-26555. 


\begin{abstract}
The Wide Field and Planetary Camera 2 on board the Hubble Space Telescope has been used to obtain high-resolution images of NGC 3610, a dynamically young elliptical galaxy in a group environment. These observations supersede shorter, undithered HST observations where an intermediate-age population of globular clusters was first discovered. The new observations show the bimodal color distribution of globular clusters more clearly, with peaks at $V-I=0.95$ and 1.17. The luminosity function of the blue, metal-poor population of clusters in NGC 3610 turns over, consistent with a Gaussian distribution with a peak $M_{V} \approx-7.0$, similar to old globular-cluster populations in elliptical galaxies. The red, metal-rich population of clusters has a luminosity function which is more extended toward both the bright and faint ends, as expected for a cluster population of intermediate age. It is well fit by a power law $\phi(L) d L \propto L^{\alpha} d L$, with an exponent of $\alpha=-1.78 \pm 0.05$, or $\alpha=-1.90 \pm 0.07$ when corrected for observational scatter. A Kolmogorov-Smirnov test confirms the significant difference between the luminosity functions of the red and blue clusters, with a probability of less than $0.1 \%$ that they come from the same population. A comparison with the Fall \& Zhang cluster disruption models shows marginal agreement with the observed data when comparing both the luminosity functions and the mean color distributions, although there are differences in detail. In particular, there is no clear evidence of the predicted turnover at the faint end, although deeper observations will be required to make a definitive test. A by-product of the analysis is the demonstration that, at any given metallicity, the peak of the luminosity function should remain nearly constant from $1.5-12$ Gyr, since the effect of the disruption of faint clusters is almost perfectly balanced by the fading of the clusters. This may help explain the apparent universality of the peak of the globular cluster luminosity function.
\end{abstract}

Key Words: galaxies: globular clusters, galaxies: interactions, galaxies: elliptical, galaxies: individual (NGC 3610) 


\section{INTRODUCTION}

Until recently, all globular clusters were considered to be among the oldest objects in the universe, and various theories were developed to explain their formation some 15 Gyr ago. However, the discovery of young massive compact clusters in merging and starbursting galaxies (e.g., Holtzman et al. 1992, 1996; Whitmore et al. 1993; Whitmore \& Schweizer 1995; Meurer et al. 1995, Schweizer et al. 1996, Zepf et al. 1999) provides a means to study the formation of what appear to be young globular clusters in the local universe.

Young merger remnants (e.g., $\lesssim 1$ Gyr) are generally easy to identify, both because of their disturbed morphologies and the burst of star formation that accompanies the interaction. Intermediate-age merger remnants (e.g., 1 - 5 Gyr) are more difficult to identify since the main body has had time to relax into a relatively symmetric configuration and the newly-formed stellar populations have had time to fade and redden (Schweizer \& Seitzer 1992). In particular, newly formed metal-rich globular clusters with ages in the range $1-2$ Gyr will have roughly the same $V-I$ colors as old metal-poor globular clusters (see Whitmore et al. 1997; hereafter Paper I), making it difficult to distinguish the two. Fortunately, the intermediate-age clusters will still be $1-2$ mag brighter than the old clusters.

Recently, a number of studies have identified intermediate-age globular clusters in several merger remnants. This helps provide the "missing link" between young merger remnants and elliptical galaxies. For example, in Paper I we estimated that NGC 3610 suffered an interaction $4 \pm 2.5$ Gyr ago. The case for NGC 1700 was less clear, although the data were compatible with an age of $\sim 4$ Gyr. A recent study by Brown et al. (2000) yields an estimated age of $2-5$ Gyr for NGC 1700 globulars. Similar results have been obtained for NGC 6702, with Georgakakis, Forbes, \& Brodie (2001) estimating an age of 2 - 5 Gyr for its red globular clusters. Currently, the best example of an early-type galaxy with intermediate-age globular clusters is probably NGC 1316, where photometric and spectroscopic observations of its red clusters yield ages of $3 \pm 0.5$ Gyr (Goudfrooij et al. 2001a,b).

The luminosity functions for clusters in young mergers, such as "The Antennae," are power laws with indices of $\alpha \approx-2$ (Whitmore et al. 1999b; Whitmore 2001). This distribution is markedly different from the peaked Gaussian profiles found for luminosity functions of old globular clusters (e.g., Figure 3 of Zhang \& Fall 1999). However, various destruction mechanisms - such as 2-body evaporation, bulge and disk shocking, and stellar mass loss - should remove the less massive, more diffuse clusters as the galaxy ages. Hence, this process may eventually yield a peaked distribution of clusters similar to what is seen for old globular clusters. Intermediate-age remnants, such as NGC 3610 (Scorza \& Bender 1990, Schweizer \& Seitzer 1992), offer the possibility of observing this process in action. While the blue, metal-poor globular clusters should have a distribution similar to old globular clusters 
in elliptical galaxies, peaking at $M_{V} \approx-7.2$ with a dispersion of $\sigma \approx 1.3$ (Whitmore 1997), the red, metal-rich clusters should extend to both brighter and fainter luminosities and may show a dropoff one or two magnitudes fainter than is seen for the old globular clusters (Fall \& Zhang 2001, Goudfrooij et al. 2001b).

In the following we adopt a Hubble Constant of $H_{0}=75 \mathrm{~km} \mathrm{~s}^{-1} \mathrm{Mpc}^{-1}$ and a mean corrected group velocity of $2251 \mathrm{~km} \mathrm{~s}^{-1}$ (Paper I), which places NGC 3610 at a distance of $30.0 \mathrm{Mpc}$, corresponding to a distance modulus of $32.39 \mathrm{mag}$. At this distance the projected scale is $1^{\prime \prime}=146 \mathrm{pc}$, and 1 pixel of the Planetary Camera $\left(0^{\prime \prime} 04554\right)$ covers $6.63 \mathrm{pc}$.

\section{OBSERVATIONS AND REDUCTIONS}

\subsection{WFPC2 Observations}

Our previous WFPC2 observations of NGC 3610 consisted of $2 \times 500$ s exposures in F555W and $2 \times 600 \mathrm{~s}$ exposures in F814W. The exposures were undithered. The new Cycle 7 observations (Proposal ID $=7468$ ), obtained on November 3, 1999, consisted of $4 \times 1200 \mathrm{~s}$ exposures in F555W and $4 \times 1400 \mathrm{~s}$ exposures in F814W. In both passbands we observed at two positions (offset from each other by $\sim 5.5$ pixels in the Planetary Camera (hereafter PC) and $\sim 2.5$ pixels in the Wide Field Camera (hereafter WF)) to permit subpixel dithering. The DRIZZLE software developed by Fruchter \& Hook (1998) was used to combine the images in each passband, with parameters set to pixfrac $=0.6$ and scale $=0.5$ (i.e., pixel size). The factor of $\sim 5$ increase in the total exposure time, the use of dithering, and the use of a new search algorithm allowed us to reach a factor of $\sim 2$ fainter in luminosity than with our 1994 observations, as can be seen by comparing the new completeness functions of Figure 1 with the corresponding functions of Figure 7 in Paper I.

\subsection{New Search Algorithm}

Our primary goal for the Cycle 7 observations of NGC 3610 was to push the detection threshold as faint as possible, in order to determine whether the luminosity function for the intermediate-age clusters continues as a power law to fainter magnitudes or turns over, as predicted by theory (e.g., Fall \& Zhang 2001). We therefore developed a new search algorithm in order to reach magnitudes as faint as possible. The first step of this algorithm uses the DAOFIND task from the daophot package (Stetson 1987) to identify potential sources down to a very faint limit ( $2 \sigma$ of background) on the $V, I$, and summed $V+I$ images. This results in a relatively large number of false detections in the bright inner regions of the galaxy. Such detections are then filtered out by requiring a $\geq 5 \sigma$ detection on a median-subtracted $V+I$ image (with aperture radii for object/inner background/outer background set to $2 / 2 / 4$ pixels on the dithered image, corresponding to $1 / 1 / 2$ pixels on the original image). The algorithm 
then performs photometry of the surviving candidate sources on both the drizzled $V$ and $I$ images. Sources are further selected by requiring a $\geq 5 \sigma$ detection in one of the two bands, and values for the concentration index (the difference in magnitude using apertures with 1 and 6 drizzled-pixel radii on the PC, or 1 and 4 drizzled pixels on the WF) between 1.4 and 2.05 on the WF images and greater than 1.8 on the PC image. This choice of concentration indices filters out both hot pixels and background galaxies (see Miller et al. 1997 for an example of how the size criteria work for undithered images). Note that there is no upper limit for the concentration index on the PC because objects very near the center have large apparent values of the index, due to the very strong galaxy background. A visual inspection and simple statistics suggest that most or all of these objects are globular clusters rather than background galaxies.

Figure 2 shows a median-subtracted F555W image of the central region of NGC 3610. The apparent warp of the central disk first described in Paper I is prominent. The red clusters with $V-I=1.025-1.5$ are circled, while the blue clusters with $V-I=0.5-1.025$ are marked by squares. The bright clusters from Table 1 are marked with larger symbols and labeled. A comparison with our earlier HST data shows that 22 of the 23 clusters in the overlap region with Figure $3 \mathrm{~b}$ of Paper I are recovered. In addition, 11 new candidate clusters are found, primarily in the inner region where our new search technique makes it possible to identify clusters in regions of higher background. These newly discovered objects include nine of the 50 brightest clusters (i.e., \#1, 4, 8, 14, 15, 16, 29, 47, and 48). Eight of these nine clusters are red, emphasizing the preponderance of bright red clusters near the center of the galaxy.

\subsection{Photometry}

Aperture corrections were determined by measuring magnitudes through various apertures for several well exposed clusters on each of the four chips. The aperture corrections for the $\mathrm{PC}$ were $-0.304 \mathrm{mag}$ in $\mathrm{V}$ and $-0.363 \mathrm{mag}$ in $\mathrm{I}$, using aperture radii for object/inner background/outer background of 6/10/16 pixels on the dithered image (i.e., 3/5/8 pixels on the original image). The corrections on the WF were $-0.312 \mathrm{mag}$ in $\mathrm{V}$ and $-0.370 \mathrm{mag}$ in $\mathrm{I}$, using aperture radii of 4/10/16 pixels on the dithered image. Small chip-to-chip corrections were also applied using the values in Table 28.1 of the HST Data Handbook (Voit 1997).

A comparison of our new photometry with the $V$ magnitudes of 40 bright clusters found earlier (Paper I, Table 4) shows a median difference of 0.10 mag, with Paper I values being fainter. The comparison is especially poor for four clusters very near the center of the galaxy, where differences in $V$ can be as large as 1 mag. Although we realized in Paper I that these clusters had large uncertainties (i.e., error estimates ranging from 0.44 to 1.05 mag), the main reason for this difference now appears to be our use of larger apertures for 
both the objects and the background in the older observations. The very strong background gradients in the inner regions and the larger sky annuli resulted in our overestimating the background in the Paper I photometry. After removing the outliers near the center of the galaxy the dispersion of the differences in $V$ is 0.09 mag, with a mean difference 0.07 (Paper I values fainter). The comparison for $V-I$ colors is better, with a mean difference of only $0.03 \mathrm{mag}$ (Paper I values redder) and a dispersion of $0.09 \mathrm{mag}$.

Completeness tests were performed for five background levels on the PC and four background levels on the WFC by using the task addstar in the DAOPHOT package. This program adds artificial objects derived by using high $\mathrm{S} / \mathrm{N}$ images of isolated clusters from the same chip. Figure 1 shows the resulting completeness functions. The completeness thresholds used in the present paper are defined as the magnitude at which only half the artificial objects are identified. Corrections for non-optimal charge transfer efficiency on the CCDs of WFPC2 were applied using the formulae by Whitmore, Heyer, \& Casertano (1999). Finally, the instrumental magnitudes were transformed to the Kron-Cousins system via the formulae by Holtzman et al. (1996).

\section{ANALYSIS}

\subsection{Color Distribution}

Figure 3 shows the new $V-I$ vs. $V$ color-magnitude diagram for NGC 3610 clusters. This diagram looks quite similar to the corresponding diagram of Paper I, but now extends to a fainter detection limit of $V \approx 26.5$ at $50 \%$ completeness. We again find a clear trend for the brighter clusters to be redder.

Figure 4 shows histograms of $V-I$ indices of candidate clusters within $50^{\prime \prime}$ from the center of NGC 3610. The left panel shows all candidate clusters brighter than $V=26.5$ while the right panel shows only those brighter than $V=25$. Note that the clusters in the PC (i.e., the area shaded black) show clearly bimodal color distributions in either case, while in the combined PC $+\mathrm{WF}$ area the brighter clusters show bimodality more cleary, presumably because of the higher signal-to-noise. This diagram was obtained as follows. All candidate clusters within $50^{\prime \prime}$ from the center and with $\sigma_{V-I} \leq 0.4$ mag were binned in 0.05-mag intervals and corrected for contamination by background objects. The latter correction was derived by selecting objects beyond 85" from the center according to the same selection criteria, smoothing their color distribution with a 7-bin triangular weight function to diminish smallnumber noise, and subtracting their surface-number density from that of the clusters within the central 50". The corrected numbers of clusters is shown as a solid line in Figure 4,

while the numbers before background correction are indicated by a dotted line. Since the background-object selection region beyond $85^{\prime \prime}$ presumably still contains some halo globular 
clusters, the correction for contamination by background objects is a maximum, and the true color distribution of globulars in NGC 3610 must lie somewhere between the solid and dotted lines (likely close to the solid lines).

The most central dozen of the selected clusters all lie close to, or within the, central warped stellar disk (Figure 2). Although all but two of these twelve clusters look round and symmetric, one might question whether some of them are disk clumps or noise of uniform color and, therefore, contribute unduly to the red peak of the bimodal color distribution. To check for this possibility, we excluded these twelve clusters by requiring that the level of the surrounding background in $V$ be less than 130 counts per pixel. The resulting color distributions looks nearly identical to Figure 4. We conclude that the very pronounced red peak of the central clusters is not an artifact of disk noise, but simply reflects the strong central concentration of this subpopulation of globulars.

The bimodality is more pronounced than in Paper 1, perhaps because of smaller measuring errors due to the longer exposures and dithered data. We used the KMM test developed by Ashman, Bird, \& Zepf (1994) to objectively estimate the probability of a bimodal distribution. A wide range of selection criteria were employed to test the robustness of the estimates. In essentially all cases the distribution was found to be bimodal, with probabilities ranging between $93 \%$ and $97 \%$ if we restrict the sample to $0.7<V-I<1.5$, $\sigma(V-I)<0.3$, and use projected distances from the center of less than $20^{\prime \prime}(\mathrm{n}=39), 30^{\prime \prime}$ $(\mathrm{n}=57)$, and $40^{\prime \prime}(\mathrm{n}=74)$. The mean values for the two peaks of the distributions fall in the ranges $V-I=0.94-0.96$ and $1.16-1.17$ for these three spatial subsamples, very similar to the values found for many elliptical galaxies (e.g., Kundu \& Whitmore 2001). Roughly $30 \%$ of the clusters appear to be members of the bluer, metal-poor population, according to the KMM test. If we extend the sample to larger projected distances the bimodality probabilities become smaller (e.g., $82 \%$ for $R<50^{\prime \prime}$ and $74 \%$ for the full field of view), presumably because of increasing contamination by background objects in the outer regions, and perhaps also because of larger photometric errors for the fainter clusters, which tend to be found in the outer regions.

A close look at Figure 3 shows that most of the clusters in the brightest $2-3$ mag range belong to the red, metal-rich population, with colors in the range $V-I=1.025-1.30$. However, at a magnitude of $\mathrm{V} \approx 25$ it appears that the dominant population is the blue, metal-poor clusters, with colors in the range $0.80-1.025$. We note that a typical value of $M_{V}$ for an old globular cluster population is -7.2 (Whitmore 1997). Hence we expect a peak in the blue population at $M_{V} \approx 25.2$, given the distance modulus of $m-M=32.39$. Near the completeness limit at $V \approx 26.5$, the metal-rich population again appears to dominate. This alternating dominance of red and blue clusters is consistent with expectations from a superposition of an old metal-poor population of globular clusters and an intermediate-age 
metal-rich population of globular clusters, if the latter has a luminosity function extending farther to both brighter and fainter magnitudes (i.e., more similar to a power law).

The statistical significance of this trend can be better quantified by computing the mean $V-I$ as a function of $\mathrm{V}$, as shown in Figure 5. We first trim the distribution to only include objects in the range $0.8<V-I<1.3$, since Figures 3 and 4 show that most candidate clusters fall in this range. Figure 5 (top) shows the $V-I$ means without any restriction on the radius. This diagram supports the first visual impression from the colormagnitude diagram: the brightest clusters at $V \approx 22$ have a mean $V-I=1.15 \pm 0.04$. The mean then migrates to $V-I=1.05 \pm 0.02$ at $V \approx 25$, and finally shifts back toward the red with $V-I=1.09 \pm 0.02$ at $V=26.25$. Excluding clusters in the range $V=24-26$, the data represent a $5 \sigma$ deviation from a value of $V-I=1.05$.

If we restrict the sample to objects within $r<20^{\prime \prime}$ (middle panel in Fig. 5), we once again find the brighter clusters with redder colors. However, objects at fainter magnitudes are missing due to the brighter completeness correction limit of the PC.

Finally, if we restrict the sample to objects within the annulus of $20^{\prime \prime}<r<50^{\prime \prime}$ (bottom panel in Fig. 5), we again find the bell-shaped curve indicative of redder mean colors at both the bright and faint ends. In addition, we are able to observe to fainter magnitudes since the objects lie on the WF chips. Here we find a continuation of the trend to redder colors, with mean $V-I=1.11 \pm 0.02$ at $V=27.2$.

\subsection{Luminosity Functions of Blue and Red Clusters}

The derivation of the luminosity function (hereafter LF) of a cluster population on a strongly varying galaxy background presents a serious challenge. The detected-cluster counts need to be corrected for incompleteness as a function of both the cluster magnitude and the local level of galaxy background. Yet, the experimentally determined completeness functions of Figure 1 (§2.3) show a strong dependence on the background level and differ sharply between the PC and WF cameras. As a result, piecing together an incompletenesscorrected luminosity function from clusters imaged by the two different cameras and lying on backgrounds of very different brightness is nearly impossible.

The problem is alleviated if one restricts oneself to clusters imaged on the WF chips. Not only are the background variations much reduced (since NGC 3610 was centered on the $\mathrm{PC}$ ), but equal detection probability is reached at $V$ magnitudes nearly 1.0 mag fainter

on the WF images than on the PC image. Therefore, we restrict our discussion of the LF of globular clusters in NGC 3610 to the LF determined from clusters imaged on Chips 
WF2 - WF4. The LF on the PC is consistent with the following results, but the low number statistics make it impossible to draw any firm conclusions.

Figure 6 shows the luminosity functions of blue and red globular clusters determined separately. The LFs corrected for contamination by background objects are shown as solid lines and are shaded black above the 50\%-completeness limits (marked by dashed vertical lines). For comparison, the LFs uncorrected for background contamination are shown as dotted lines.

These luminosity functions were determined as follows. First, candidate clusters were separated into two subsamples of blue $(0.80 \leq V-I \leq 1.025)$ and red $(1.025<V-I \leq 1.30)$ clusters. For each of these subsamples, all candidate clusters imaged on WF chips and lying within a limiting radius $R_{\lim }=50^{\prime \prime}, 65^{\prime \prime}$, or $80^{\prime \prime}$ from the center were selected, subject to the conditions that $19 \leq V \leq 28, \sigma_{V-I}<0.60 \mathrm{mag}$, and the galaxy-background level be $0.1 \leq V_{\mathrm{bkg}} \leq 100$ counts/pixel. Each cluster was then corrected for incompleteness by dividing its count of one by the completeness fraction corresponding to its $V$ magnitude and background level. This fraction itself was obtained from the functions shown in Figure 1 by bilinear interpolation. The corrected (i.e., weighted) cluster count was then added to the appropriate bin in $V$ magnitude, with bin sizes of typically 0.25 mag.

The resulting raw luminosity functions were further corrected for contamination by fore- and background objects (i.e., stars and distant galaxies). To that effect, objects were selected at distances $R_{\mathrm{bkg}}>85^{\prime \prime}$ from the center and subject to the same color, magnitude, and galaxy-background selection criteria as the objects in the inner search zones. The "luminosity functions" of these objects were determined in a similar manner as described above, then smoothed by a 7-bin triangular weighting function to diminish small-number noise, scaled to the areas of the inner search zones, and subtracted from the above raw LFs. It is these corrected LFs that are shown as solid lines (and shaded black above the 50\% completeness limits) in Figure 6.

As Figure 6 illustrates, the luminosity functions of blue and red globular clusters in NGC 3610 differ dramatically. While the LF for the blue globulars is approximately Gaussian, that for the red globulars is more nearly power-law shaped. When compared to the blue clusters, there are more red clusters both at the bright end and near the 50\%completeness limit, consistent with the mean colors discussed in $\S 3.1$ and shown in Figure 5. The LFs shown are for 205 candidate globular clusters (64 blue, 141 red) on the WF chips out to $R_{\text {lim }}=80^{\prime \prime}$. The same shape difference is observed for the LFs of clusters out to $R_{\lim }=50^{\prime \prime}$ and $65^{\prime \prime}$, though with more noise due to smaller cluster numbers.

The statistical significance of this result can be better quantified by performing a 
Kolmogorov-Smirnov test on the populations of red globular clusters and blue globular clusters, as defined above. Figure 7 shows the results based on the completeness- and background-corrected luminosity functions from Figure 6. The top panel shows the fraction of each distribution as a function of $V$ magnitude for the clusters on the WF chip. The value of the largest discrepancy is shown on the figure, as is the probability that the two populations come from the same distribution (i.e., only a $1.1 \%$ chance). We note that the nature of the trend, with more red globular cluster's at both the bright and faint ends, tends to cancel out the value of the largest discrepancy. The two lower panels in Figure 7 show the results if we break the cumulative distributions in two at the cross-over point, $V \approx 25$. The largest fractional discrepancies are now $\approx 0.4$. While the small number of clusters in the bright part of the WF sample results in a relatively large value of the probability $(2.1 \%)$, the faint sample now shows a very small probability $(0.09 \%)$ that the red and blue globular clusters are drawn from the same sample.

\subsection{Comparison with the Fall \&5 Zhang (2001) Cluster Disruption Models}

Fall and Zhang (2001) have developed models for the disruption of globular clusters, which include two-body relaxation, gravitational shocks, and stellar evolution. Figure 4 in Fall \& Zhang (2001) demonstrates how an initial power-law or Schechter mass function will evolve toward a peaked distribution, typical of an old globular cluster population. As the fainter clusters are destroyed, the peak of the distribution shifts toward larger mass. However, the luminosities of the clusters fade at almost exactly the same rate (e.g., see Figure 13 in Paper I), hence compensating for the effect. The net result is that, at a given metallicity, the peak luminosity remains almost constant with time in the range $1.5-12$ Gyr. This may help explain the apparent universaility of the globular cluster luminosity function, as demonstrated by various studies (e.g., Harris, 1991)

Figure 8 demonstrates this effect. We combined the Fall \& Zhang (2001) model that employs a Schechter initial mass function (i.e., the bottom left panel in their Figure 4) and the prediction for fading from the Bruzual \& Charlot (1996, unpublished) stellar evolution models in order to predict the relative shifts between the peaks of the various distributions. Solar metallicity is used for the young and intermediate-age clusters while 0.02 solar metallicity is used for the old (12 Gyr) population. The models are normalized at $V=25$. The top panel shows the luminosity function on a log scale while the bottom panel shows it on a linear scale. The predicted peaks for the 1.5, 3, and 6 Gyr models are essentially identical. The only reason for the shift in the peak of the 12 Gyr old population is the lower metallicity. If we were to use solar rather than 0.02 solar metallicity for the 12 Gyr population, we would find that the peaks of the distributions vary by only $\sim 0.10$ mag over the full range from 1.5 -12 Gyr. 
The tail of the predicted LF at the faint end is nearly identical for all three intermediate ages, but the bright end shows some variation. In particular, the 1.5 Gyr population is predicted to have more bright clusters than the 12 Gyr old clusters while the 6 Gyr population is predicted to have fewer. Based on this fact alone it would appear that the intermediate-age clusters in NGC 3610 would fall in the range 1.5 - 3 Gyr. We note that the turnover in the 12 Gyr population is predicted to be about 1.5 magnitudes brighter than our completeness limit of $\sim 26.6$, but the predicted turnover in the intermediate-age population is only $\approx 0.5$ magnitudes brighter. Figure 6 does not show any clear evidence for a turnover of the red population out to the completeness limit, although there is a hint that it may flatten out beyond this point. Deeper observations will be required to make a definitive test, however.

While the young clusters in recent mergers appear to have roughly solar metallicity (e.g., Schweizer \& Seitzer, 1998), the metallicity for older remnants is more uncertain, and may tend to be lower since the merger event happened further in the past. If we adopt 0.4 solar for the metallicity of the $1.5-6$ Gyr populations we would find that the peak luminosities move toward brighter magnitudes by $\sim 0.3 \mathrm{mag}$, reducing only slightly the difference between the metal-poor 12 Gyr population and the metal-rich younger populations.

These models can also be used to predict how the values of mean $(V-I)$ change with magnitude. Figure 9 shows the predicted mean colors, based on the models in Figure 8. We find the same qualitative behavior as shown by our data for NGC 3610 in Figure 5, with redder colors for both bright and faint clusters and a mean color $\approx 1.05$ in the intermediate range. The best agreement appears to be with the 1.5 Gyr models. However, we note that the bluest mean colors are predicted to be in the range $V \approx 23-24$, while the observed peak is $V \approx 25.4$. One possible explanation for this difference might be the fact that the models assume a zero-age burst of cluster formation, while there is good evidence - both observationally (Whitmore et al. 1999b) and theoretically (Mihos et al. 1993) — that clusters can be produced for roughly half a billion years during a merger.

\subsection{Fits and Simulations of the Blue and Red Populations}

Figure 10 shows a Gaussian fit to the blue clusters (upper panel) and a variety of power-law and Fall \& Zhang (2001) model fits to the red clusters (lower panel). The Gaussian fit to the blue population yields a peak at $V=25.44 \pm 0.10$ and a width $\sigma=0.66 \pm 0.19$. This corresponds to a value of $\mathrm{M}_{V}=-6.95$, similar to the value found for normal elliptical galaxies (e.g., Whitmore 1997 finds $\mathrm{M}_{V}=-7.21 \pm 0.26$ ). We note, however, that the value of $\sigma$ is considerably smaller than typically found for giant elliptical galaxies (e.g., $1.1-1.5$, according to Kundu \& Whitmore 2001). Fixing $\sigma$ to these values yields peaks in a range from $V=25.61 \pm 0.15$ (using $\sigma=1.1$ ) to $V=26.04 \pm 0.3$ (using $\sigma=1.5$ ). However, as 
Figure 10 shows, even the fit with $\sigma=1.1$ is clearly too wide, hence we adopt the original value of $V=25.44$ as our best-fit.

The lower panel of Figure 10 includes a best-fit power law $\phi(L) d L \propto L^{\alpha} d L$, with an exponent of $\alpha=-1.78 \pm 0.05$ (thick solid line), and a set of Fall \& Zhang (2001) models ranging from zero age to 12 Gyr. The best fitting Fall \& Zhang model is the 1.5 Gyr model (dotted line). The fits to the 0 and 3 Gyr models are quite similar while the fits to the 6 and 12 Gyr population are clearly unreasonable. Hence, the simple power law provides the best overall fit to the red population, partly because the bright end slopes of the Fall \& Zhang models using the Schechter initial mass distribution appear to be a bit too steep.

Fall \& Zhang (2001) also calculated models using a shallower power-law of index -2 for the initial mass distribution (i.e., the top left diagram in their Figure 4). Figure 11 shows the fits to the data using these models. While the agreement is better at the bright end, the fit on the faint end is essentially unchanged. In particular, there is no clear evidence for the predicted flattening. However, the current data is not deep enough to make a definitive test.

Goudfrooij et al. (2001a) claimed that the luminosity function for the red clusters in the 3 Gyr old merger remnant NGC 1316 has a shallower slope, with $\alpha=-1.2 \pm 0.3$. However, a comparison of their Figure 13a with our Figure 6 shows that the two luminosity functions are actually quite similar. It appears that an error was made in the calculation of $\alpha$. Goudfrooij (2002; private communication) has corrected the problem and now finds $\alpha \approx-1.7 \pm 0.1$ for the red clusters in NGC 1316, which is similar to the value we find in NGC 3610. We note that the fits are actually computed by using the log Number vs. $V$ correlation (e.g., the bottom plot in Figure 10) and then converting it to the form $\phi(L) d L \propto L^{\alpha} d L$ via the formula $\alpha=2.5 \times \beta+1$, where $\beta$ is the slope in the $\log$ Number vs. $V$ diagram.

One of the difficulties of extracting the luminosity function for the red and blue clusters is the necessity of defining color bins which are smaller than the observational uncertainies at faint magnitudes. For example, this means that an intrinsically blue cluster may fall outside the $0.80<V<1.025$ range simply due to large observational measuring errors. Conversely, an intrinsically red cluster may be counted as a blue cluster because it is scattered into the blue bin.

We can correct for this effect by using simulations similar to those developed in Paper I. These are designed to approximately match the color-magnitude diagram, the total luminosity function, and the color histogram for the brighter clusters. We then use these simulations to determine correction fractions for the blue and red populations as a function of magnitude. 
For the blue clusters we adopt a Gaussian mass function with parameters designed to approximately match the fit shown in the upper panel of Figure 10, an age of 15 Gyr, and a metallicity $[\mathrm{Fe} / \mathrm{H}]=-1.3$. This results in a mean value of $V-I=0.95$. For the red clusters we adopt a power-law mass function with $\alpha=-1.8$, an age of $6 \mathrm{Gyr}$, and metallicity $[\mathrm{Fe} / \mathrm{H}]$ $=0.0$. This results in a mean value of $V-I=1.17$. We scale the observational uncertainies so that they mimic the scatter in the color-magnitude diagram and color histogram. The resulting age determination of 6 Gyr is consistent with our estimate of $4 \pm 2.5$ Gyr from Paper I, but is larger than the estimate of 1.5 Gyr based on a comparison with the Fall \& Zhang (2001) models. However, we note that the uncertainties in both estimates are roughly a factor of two, hence there is no serious discrepancy in the comparison.

Figure 12 shows the color magnitude diagram for the simulation. This demonstrates how the data points for some blue and red clusters are scattered into regions outside the appropriate color bins. We note that Figure 12 looks somewhat different than Figure 3 for two reasons. First, the open and filled symbols in Figure 12 are the intrinsic blue and red clusters while in Figure 3 they are the clusters on the PC and WF. Second, Figure 3 includes background objects that are not included in the simulation.

Figure 13 shows both the intrinsic (dotted line) and apparent (solid line) luminosity functions for the simulation from Figure 12. This demonstrates how observational scatter can affect our determinations of the LFs for the blue and red populations. In particular, the scatter results in an underestimate in the slope of the power law for the red population, although the effect is relatively minor (i.e., the value of $\alpha$ changes from $1.77 \pm 0.06$ to 1.90 $\pm 0.07)$.

\section{SUMMARY}

New, deeper, dithered observations using the WFPC2 on board the Hubble Space Telescope have been used to extend our study of NGC 3610, an elliptical galaxy that is a likely intermediate-age merger remnant. Our primary results are the following.

1. The inner globular clusters show a clear bimodal color distribution, with peaks at $V-I=0.95$ and 1.17. The KMM test developed by Ashman, Bird, \& Zepf (1994) yields probabilities for bimodality ranging between $93 \%$ and $97 \%$ if we restrict the sample to have projected distances from the center of less than $20^{\prime \prime}(\mathrm{n}=39), 30^{\prime \prime}(\mathrm{n}=57)$, and $40^{\prime \prime}(\mathrm{n}=74)$. Bimodality probabilities become smaller in the outer regions (e.g., $74 \%$ for the full field of view), presumably because of increasing contamination by background objects and larger photometric errors for fainter objects. 
2. The luminosity function of the blue, metal-poor population (i.e., $0.8<V-I \leq$ 1.025) of clusters in NGC 3610 turns over, with a peak $M_{V} \approx-7$, similar to values typical for old globular-cluster populations in elliptical galaxies.

3. The red, metal-rich population $(1.025 \leq V-I<1.3)$ of clusters has a luminosity function which is more extended toward both the bright and faint ends, as expected for an intermediate-age cluster population. This is evident in both the luminosity function and the distribution of mean colors. The red population is well fit by a power law $\phi(L) d L \propto L^{\alpha} d L$, with an exponent of $\alpha=-1.78 \pm 0.05$, or $\alpha=-1.90 \pm 0.07$ when corrected for observational scatter using a simulation of the data. A Kolmogorov-Smirnov test indicates that the probability that the red and blue clusters come from the same population in NGC 3610 is less than $0.1 \%$.

4. A comparison with the Fall \& Zhang (2001) cluster disruption models shows marginal agreement with the observed data when comparing both the luminosity functions and the mean color distributions. For example, the model predicts the bluest mean colors should occur in the range $V \approx 23-24$ while the observed peak occurs at $V \approx 25.4$. The models also predict a flattening and turnover of the peak in the LF of the red clusters at $V$ $\approx 26$. While there is no clear evidence of the predicted turnover, deeper observations will be required to make a definitive test.

5. A by-product of the analysis is the demonstration that, at any given metallicity, the peak of the luminosity function should remain nearly constant from 1.5 - 12 Gyr, since the effect of the disruption of faint clusters is almost perfectly balanced by the fading of the clusters. This may help explain the apparent universality of the peak of the globular cluster luminosity function.

We thank Paul Goudfrooij and Michael Fall for useful discussions. This work was supported by NASA grant GO-07468.01-A. Partial support by the NSF through Grant AST 9900742 to F.S. is also gratefully acknowledged. Partial support to B.W.M. was provided by the Gemini Observatory, which is operated by the Association of Universities for Research in Astronomy, Inc., on behalf of the international Gemini parthership of Argentina, Australia, Brazil, Canada, Chile, the United Kingdom and the United States of America. AK acknowledges support from NASA LTSA grant NAG5-11319, and from NASA grant HST-AR-08755.04-A. 


\section{REFERENCES}

Ashman, K. M., Bird, C. M., \& Zepf, S. E. 1994, AJ, 108, 2348

Brown, R. J. N., Forbes, D. A., Kissler-Patig, M., \& Brodie, J. P. 2000, MNRAS, 317, 406

Burstein, D. \& Heiles, C. 1984, ApJS, 54, 33

Fall, S. M. \& Zhang, Q. 2001, ApJ, 561, 751

Fruchter, A.S. and Hook, R.N., 1998, astro-ph/9808087

Georgakakis, A. E., Forbes, D. A. \& Brodie, J. P. 2001, MNRAS, 324, 785

Goudfrooij, P., Mack, J., Kissler-Patig, M., Meylan, G., \& Minniti, D. 2001a, MNRAS, 322, 643

Goudfrooij, P., Alonso, M. V., Maraston, C., \& Minniti, D. 2001b, MNRAS, 328, 237

Harris, W. E. 1991, ARAA, 29, 543

Holtzman, J. et al. (the WFPC2 team) 1992, AJ, 103, 691

Holtzman, J. A. et al. (the WFPC2 team) 1996, AJ, 112, 416

Kundu, A. \& Whitmore, B. C. 2001, AJ, 121, 295

Meurer, G. R., Heckman, T. M., Leitherer, C., Kinney, A., Robert, C., Garnett, D. R. 1995, AJ, 110, 2665

Mihos, J. C., Bothun, G. D., \& Richstone, D. O. 1993, ApJ, 418, 82

Miller, B. W., Whitmore, B. C., Schweizer, F., \& Fall, S. M. 1997, AJ, 114, 2381

Schweizer, F., Miller, B., Whitmore, B. C., \& Fall, S. M. 1996, AJ, 112, 1839

Schweizer, F. \& Seitzer, P. 1992, AJ, 104, 1039

Schweizer, F. \& Seitzer, P. 1998, AJ, 116, 2206

Szorza, C. \& Bender, R. 1990, A\&A,235, 49

Stetson, P. B. 1987, PASP, 99, 191

Voit, M. 1997, HST Data Handbook (Space Telescope Science Institute, Baltimore)

Whitmore, B. C. 1997, in The Extragalactic Distance Scale, ed. M. Livio, M. Donahue, N. Panagia (STScI, Baltimore), 254.

Whitmore, B. C. 2001, astro-ph/0012546 
Whitmore, B. C., Heyer, I., \& Casertano, S. 1999, PASP, 111, 1559

Whitmore, B. C., Miller, B. W., Schweizer, F., \& Fall, S. M. 1997, AJ, 114, 1797 (Paper I)

Whitmore, B. C., \& Schweizer, F. 1995, AJ, 109, 960

Whitmore, B. C., Schweizer, F., Leitherer, C., Borne, K., \& Robert, C. 1993, AJ, 106, 1354

Whitmore, B. C., Zhang, Q., Leitherer, C., Fall, S. M., Schweizer, F., \& Miller, B. W. 1999, AJ, 118, 1551

Zepf, S. E., Ashman, K. M., English, J., Freeman, K. C., \& Sharples, R. M. 1999, AJ, 118, 752

Zhang, Q. \& Fall, M., 1999, ApJ, 527, L81

This preprint was prepared with the AAS LATEX macros v4.0. 


\section{FIGURE CAPTIONS}

Figure 1 - Completeness curves as determined from artificial star experiments for different background levels on the PC and WF. The background levels are given in units of DN (Data Number).

Figure 2 - Median-subtracted image in F555W showing the inner region of NGC 3610. Squares are blue clusters while circles are red clusters. Larger symbols are used for the 50 brightest clusters.

Figure $3-V-I$ vs. $V$ color-magnitude diagram for cluster candidates in NGC 3610. Open circles are used for the objects on the PC while filled circles are used for the WF.

Figure 4 - Color distributions of NGC 3610 candidate clusters within 50" from the center; all clusters (solid lines) and clusters on PC chip only (shaded black). The dotted lines show clusters before correction for background-object contamination. Left panel shows all candidate clusters brighter than $V=26.5$, while right panel shows only those brighter than $V=25$. Note that the clusters in the $\mathrm{PC}$ area show clearly bimodal color distributions in either case, while in the combined PC + WF area the brighter clusters show bimodality more cleary.

Figure 5 - Mean values of $V-I$ for clusters in the range $0.8<V-I<1.3$ as a function of $V$ for various radial ranges in NGC3610. In all cases the smallest mean value is near $V=25$, with higher values on both the bright and faint ends. This shows that the distribution of red globular clusters is more extended (i.e., is more like a power law) than the distribution of blue globular clusters (which is more like a Gaussian).

Figure 6 - Luminosity functions of blue (left) and red (right) globular clusters in NGC 3610 within $80^{\prime \prime}$ from the center. The $50 \%$ completeness limits (vertical dashed) mark the boundary up to which the LFs can be trusted. Areas shaded dark mark background-corrected LFs, dotted lines the uncorrected LFs. Notice the nearly lognormal shape of LF for blue globulars and the nearly power-law LF for red globulars.

Figure 7 - Kolmogorov-Smirnov tests comparing the populations of blue globular cluster (0.8 $<V-I \leq 1.025)$ and red globular cluster $(1.025<V-I \leq 1.3)$. The largest discrepancies are listed along with the probability that the two samples come from the same population. The cross-over near $V=25$ tends to cancel out the differences in the two populations, hence the bottom two panels break the cumulative distribution in half. In all cases we find strong evidence that the two populations are statistically different. 
Figure 8 - Predicted luminosity functions (log in the top panel; linear in the bottom panel) based on a combination of the Fall \& Zhang (2001) cluster disruption models and the Bruzual \& Charlot (1996) population synthesis models. Solar metallicity is assumed for the young and intermediate-age clusters while 0.02 solar is assumed for the 12 Gyr population. Note that the fading predicted by the BC96 models almost exactly cancels out the increase in the mean mass predicted by the Fall and Zhang models, resulting in nearly identical distributions at the faint end for the 1.5, 3, and 6 Gyr models.

Figure 9 - Predicted values of mean $V-I$ for the models shown in Figure 8. Note that while the 1.5 and 3 Gyr models have the same qualitative behavior as shown in Figure 5, the predicted position of the peak is bluer than found for the observations of NGC 3610 .

Figure 10 - Fits to the luminosity functions of blue (upper) and red (lower) globular clusters in NGC 3610. The upper panel shows our best-fit Gaussian (thick solid line; $V=25.44 \pm 0.10$, $\sigma=0.66 \pm 0.19)$ for the blue clusters, along with a fit with $\sigma=1.1$ (dotted line), which is clearly unreasonable. The lower panel shows a best-fit power law (thick solid line; slope $=-1.78 \pm 0.05$ ), and a range of Fall \& Zhang (2001) models using Schechter initial mass functions and ages ranging from zero age (thin solid line) to 12 Gyr (3dot - dash line). The best fitting Fall \& Zhang model is the 1.5 Gyr model (dotted line). The 0 and 3 Gyr models give similar results, while the 6 and 12 Gyr models result in unreasonable fits.

Figure 11 - Same as Figure 10, but using Zhang \& Fall (2001) models with a power-law distribution for the initial mass function.

Figure 12 - A simulation of the $V-I$ vs. $V$ color-magnitude diagram for cluster candidates in NGC 3610. Filled circles are intrinsically red clusters while open circles are intrinsically blue clusters. Note how observational measuring errors can scatter the data points into the wrong color bins. See text for details about the simulation parameters.

Figure 13 - The intrinsic (dotted line) and apparent (solid line) luminosity functions for the simulation shown in Figure 12. This shows that observational scatter can result in an overestimate in the value of $V$ for the peak of the blue population and an underestimate in the slope of the power law for the red population. 

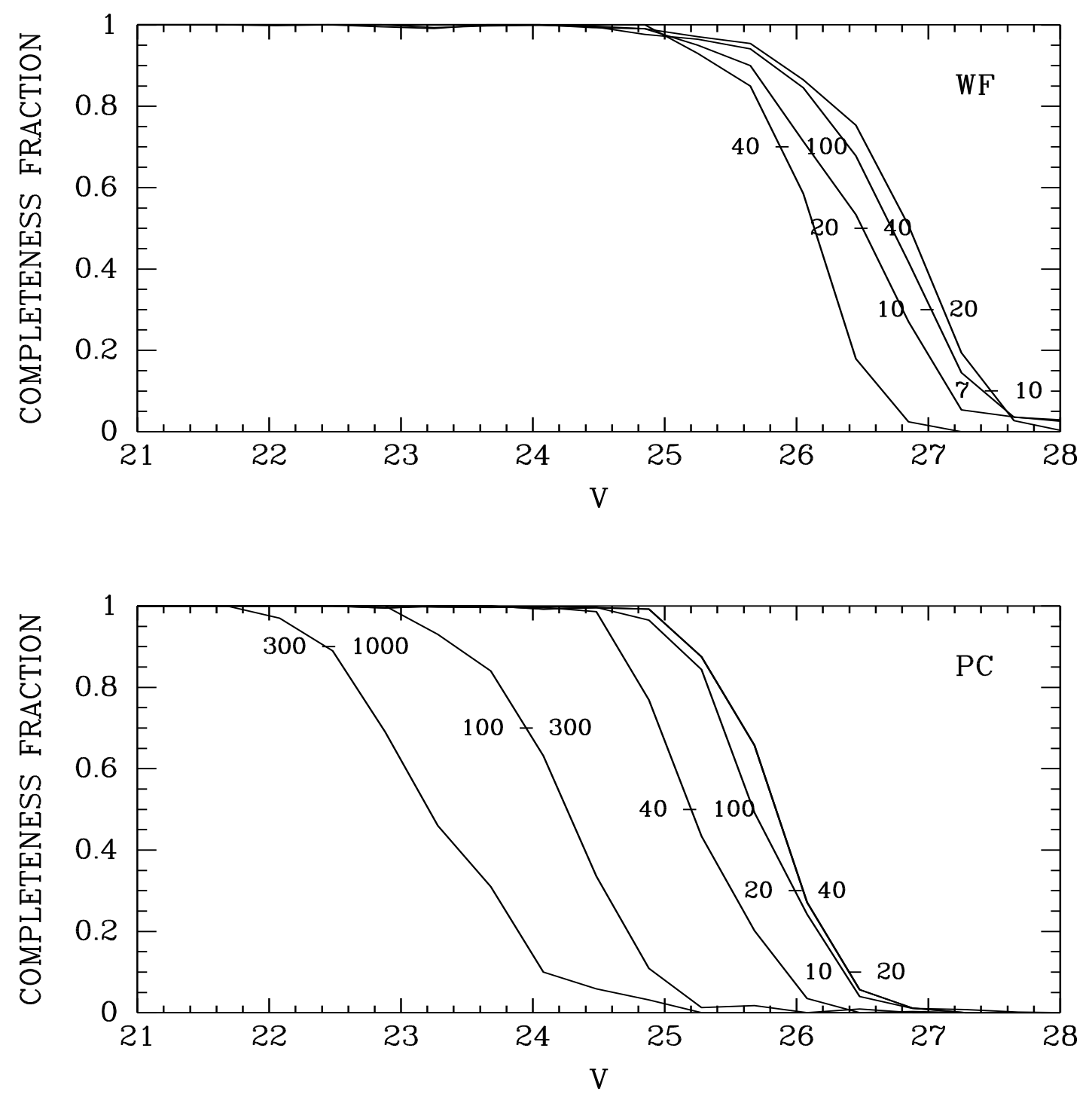

Fig. 1.- 


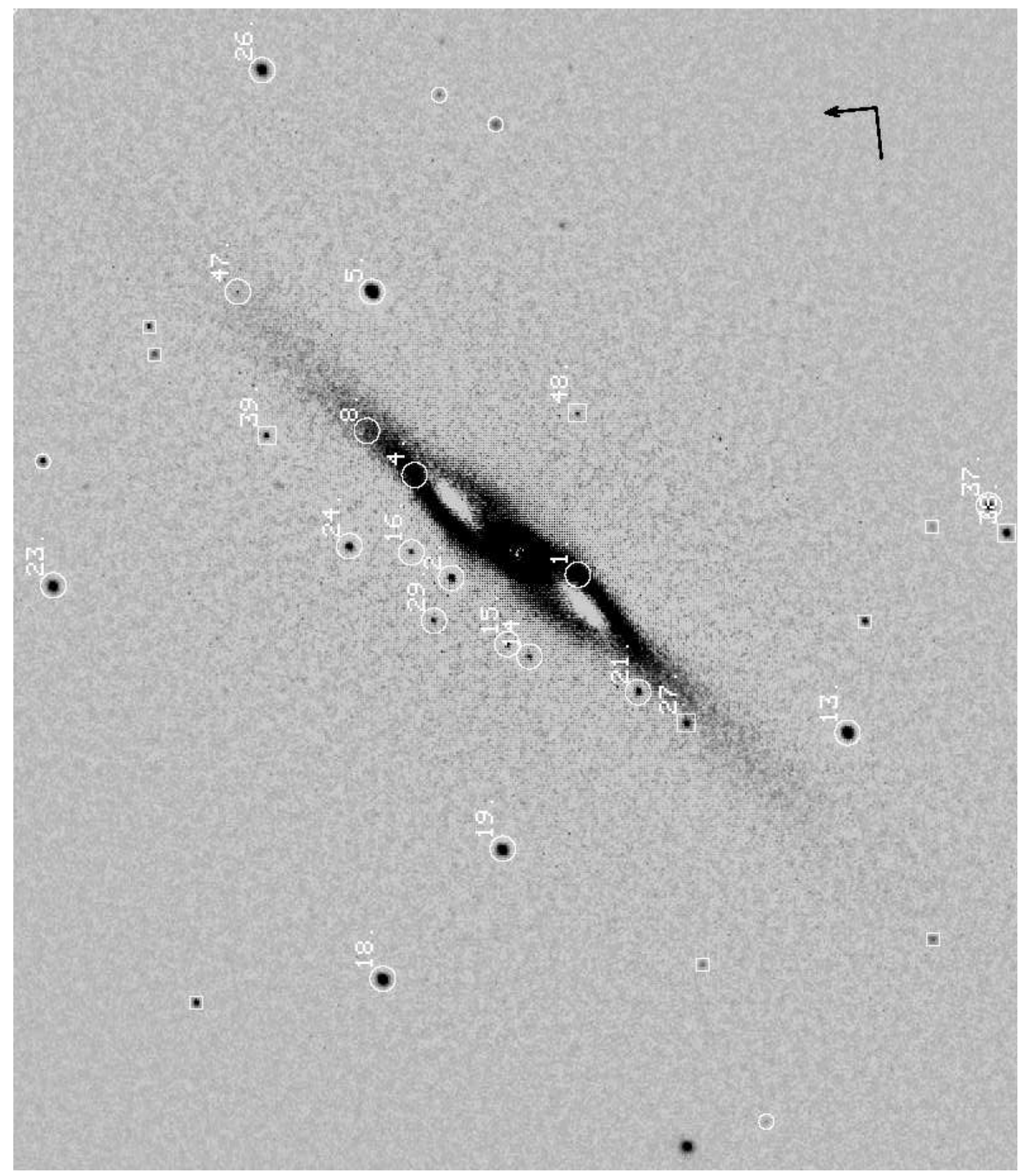

Fig. 2.- 


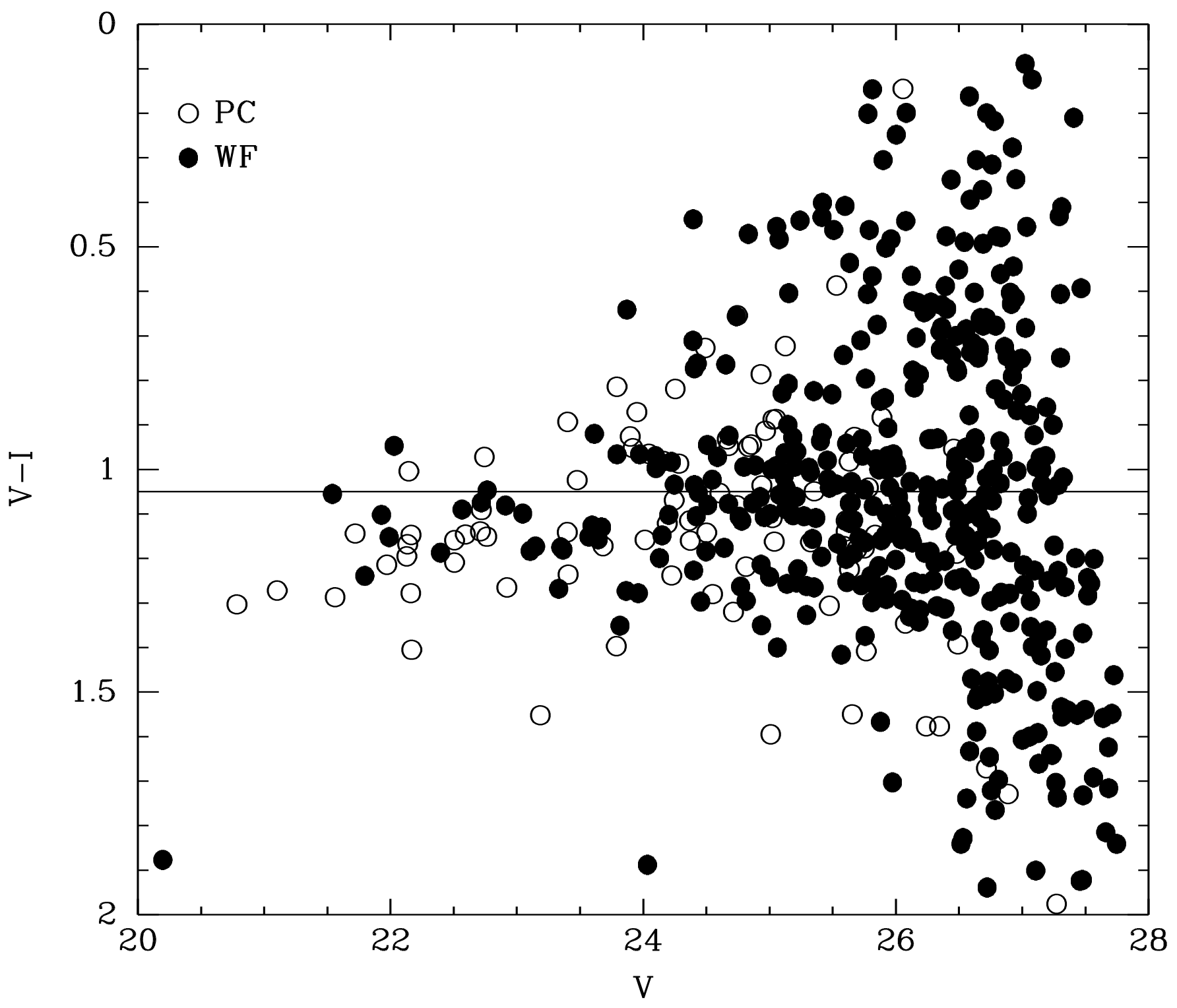

Fig. 3.- 


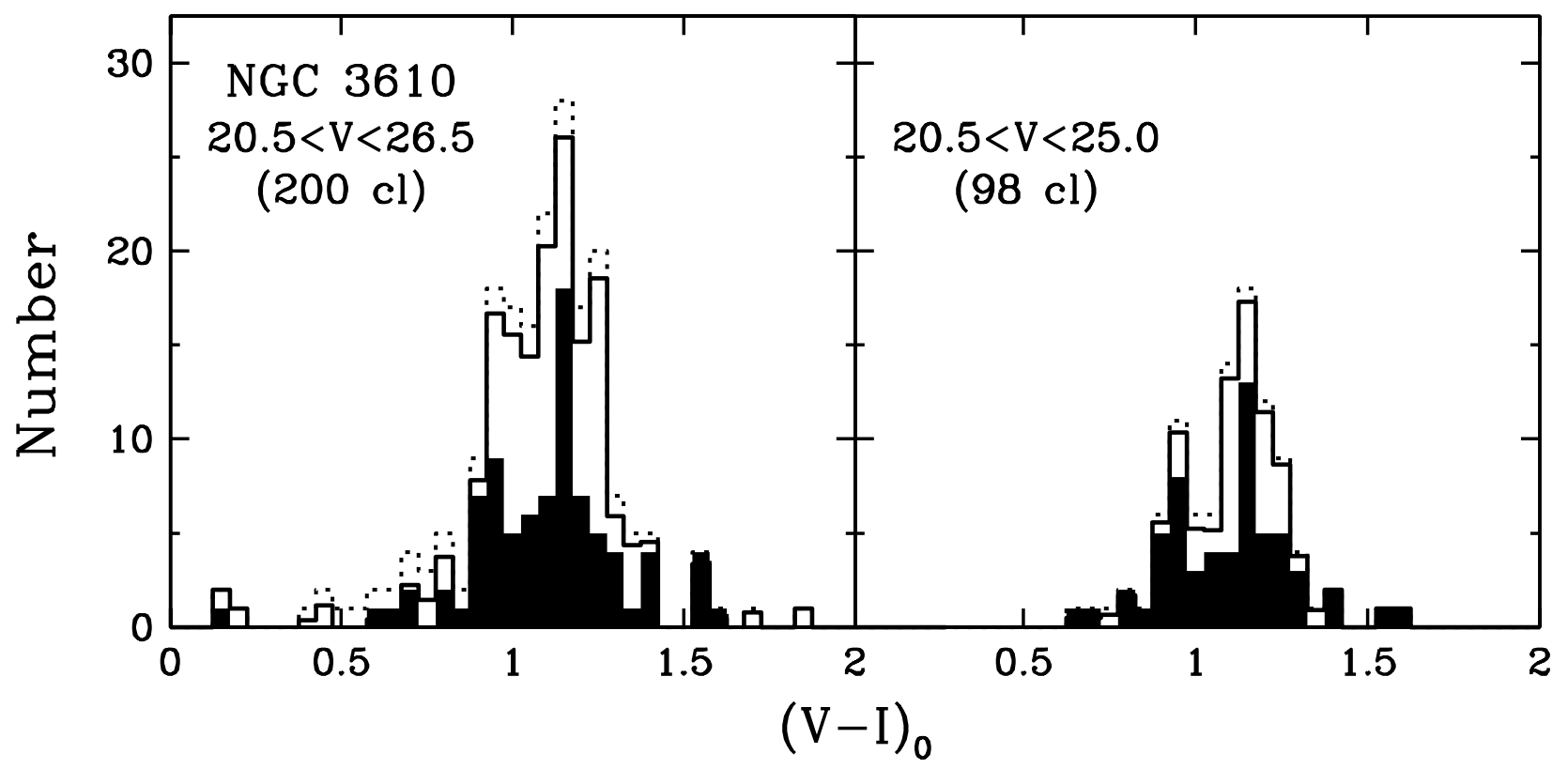

Fig. 4.- 

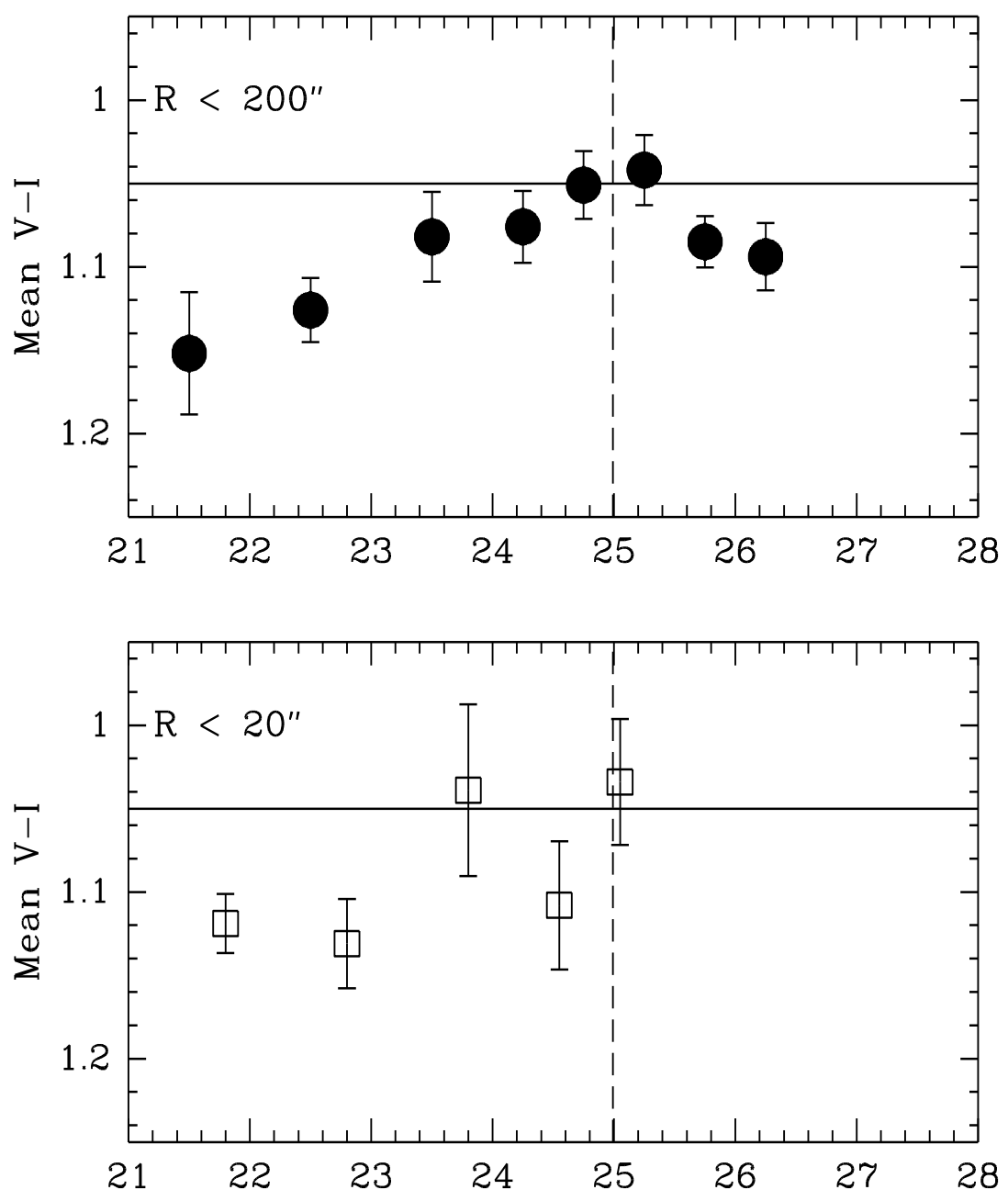

Fig. 5.-

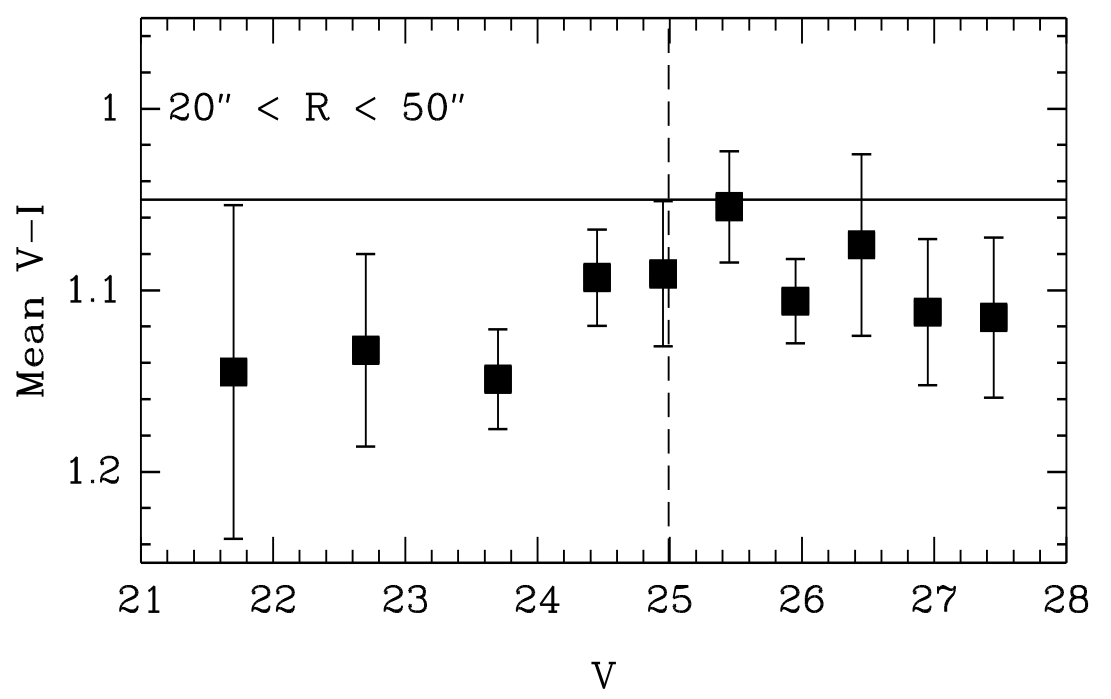




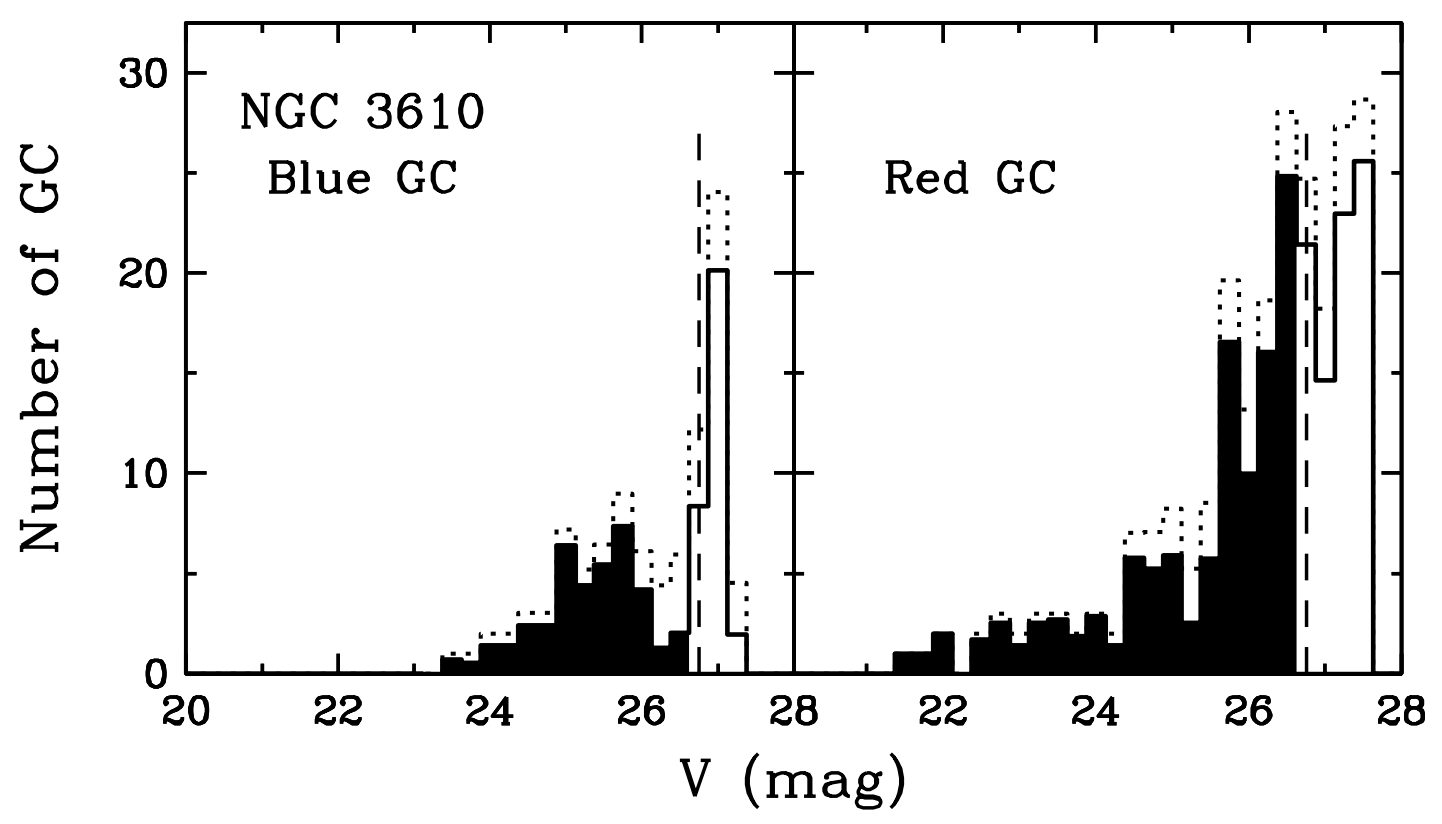

Fig. 6.- 

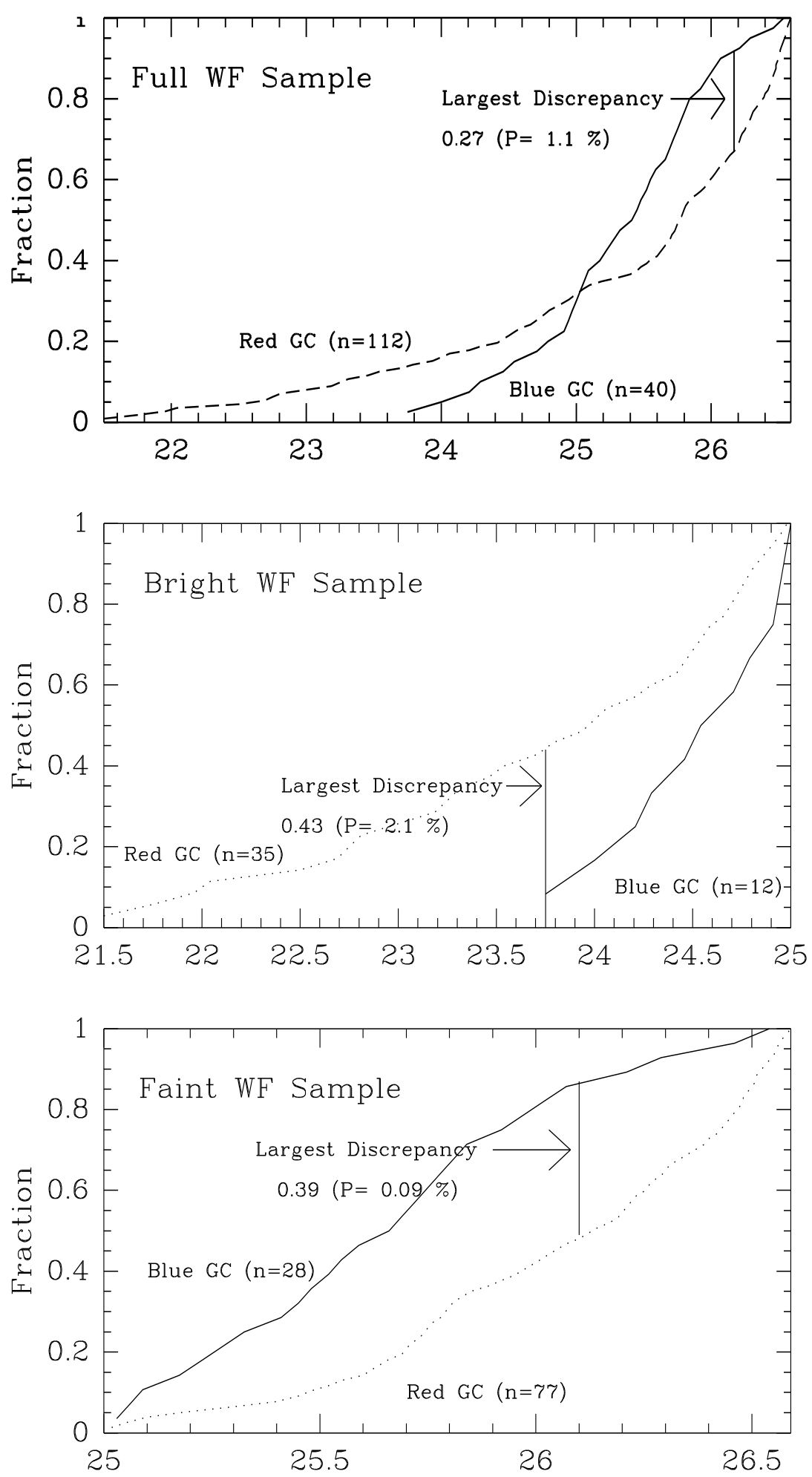

Fig. 7.- 

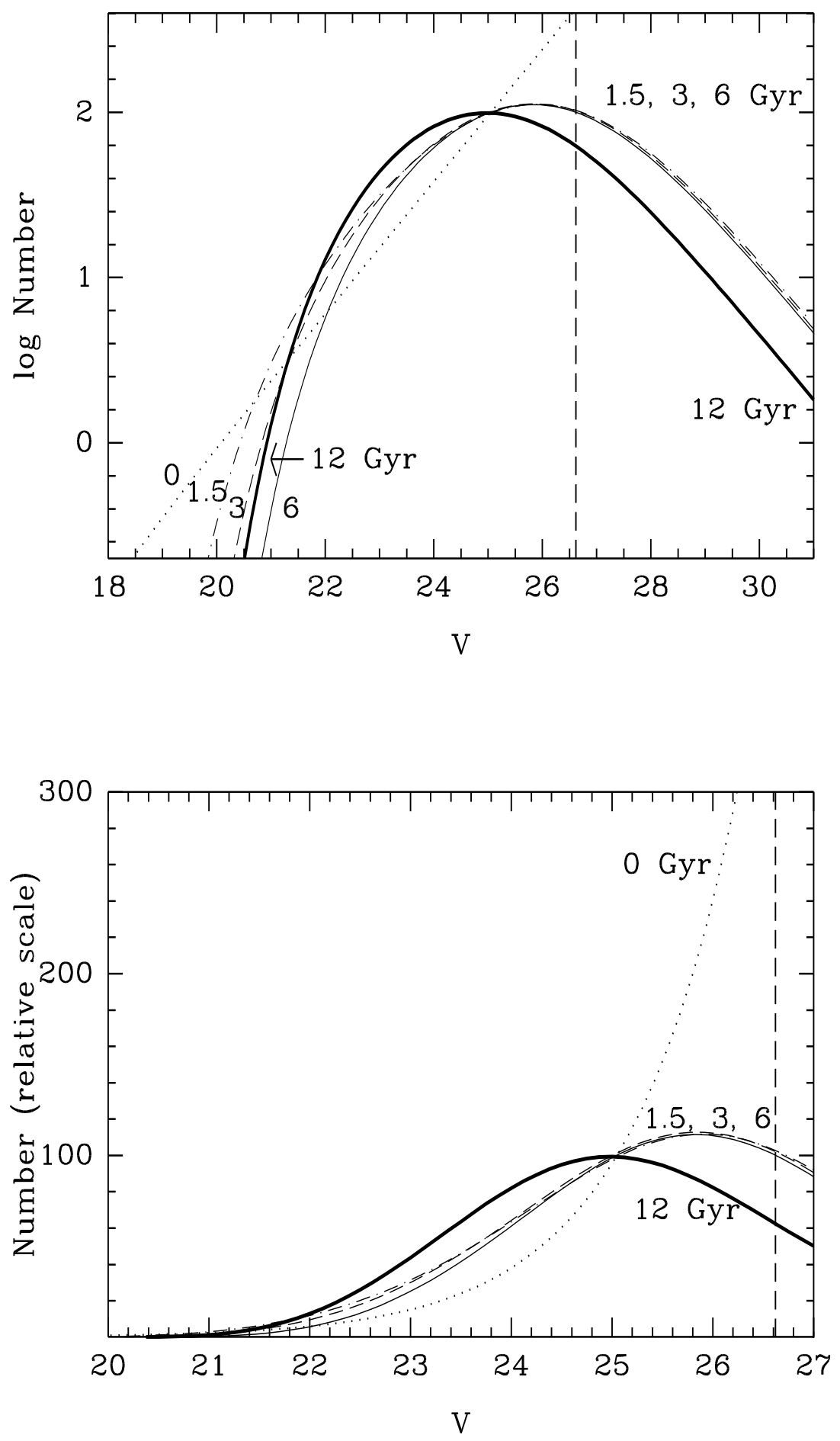

Fig. 8.- 


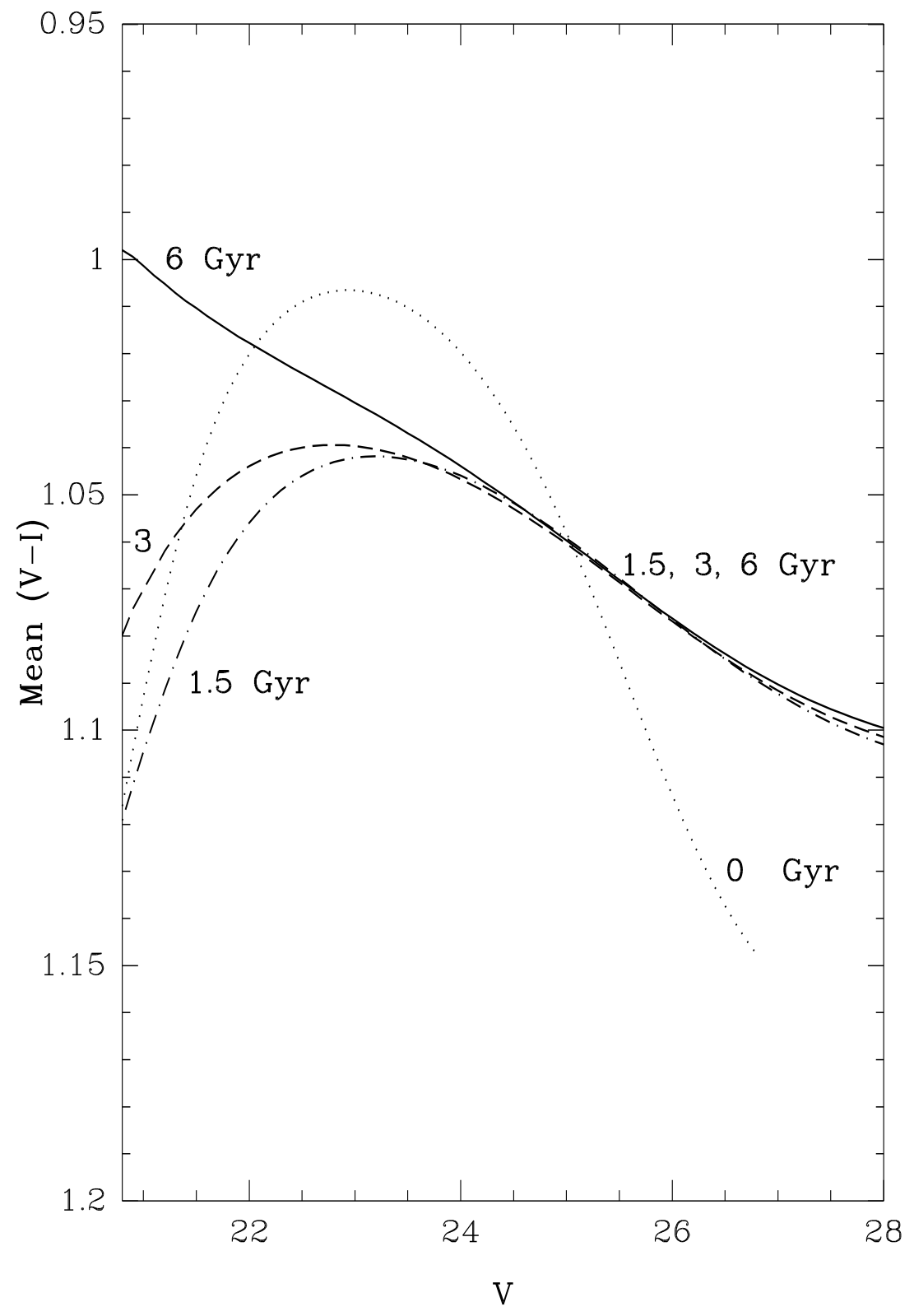

Fig. 9.- 


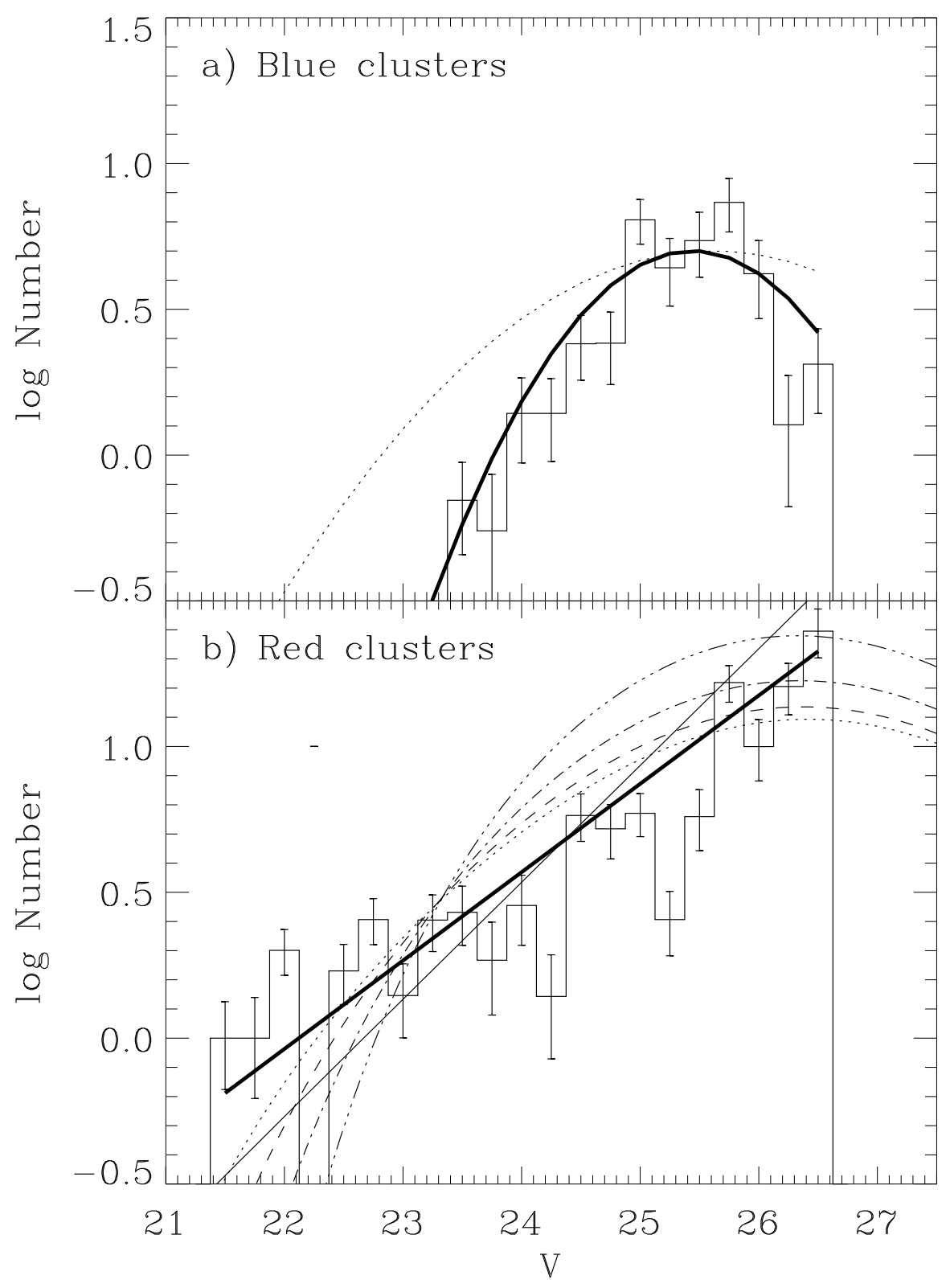

Fig. 10.- 


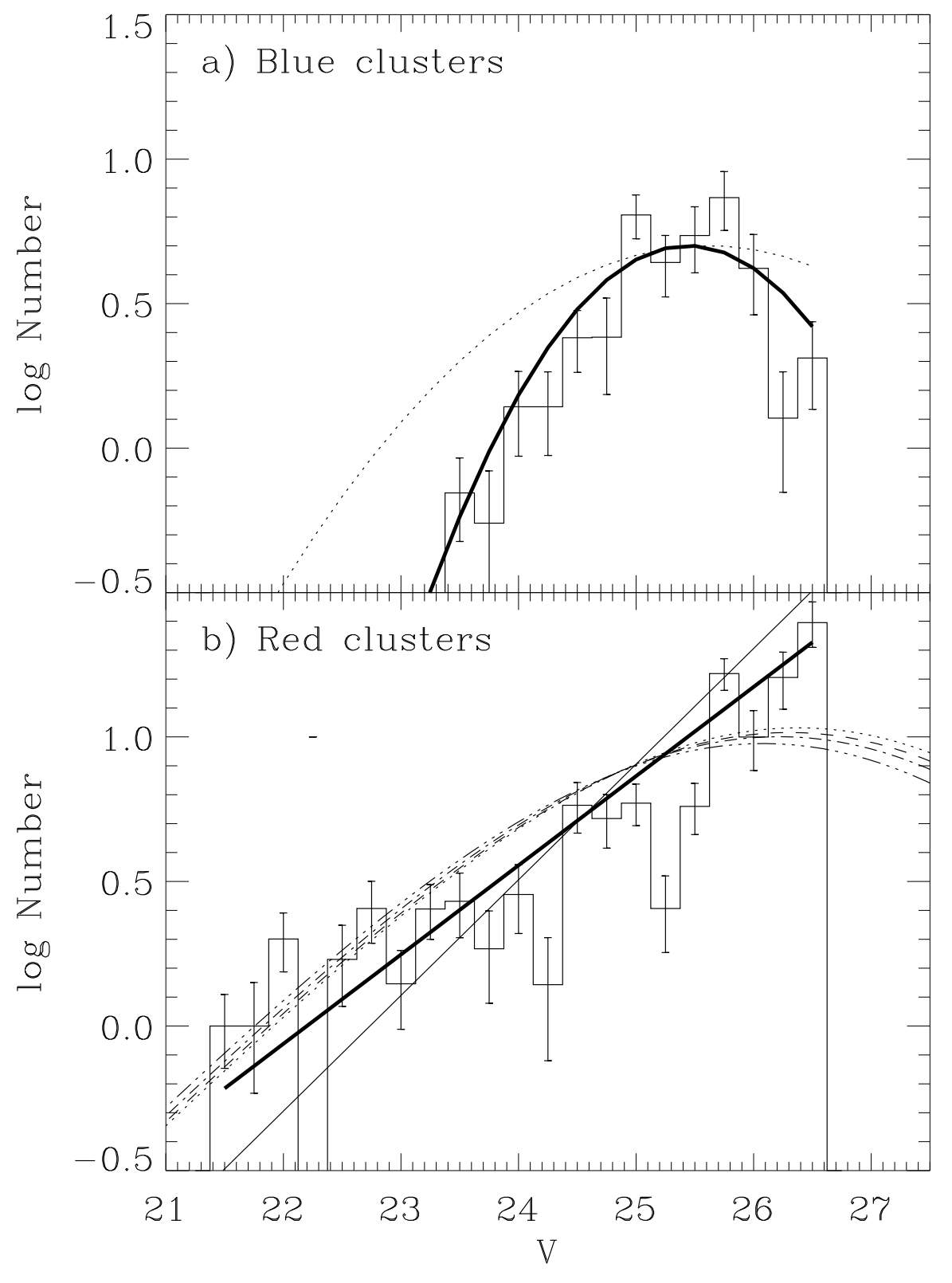

Fig. 11.- 


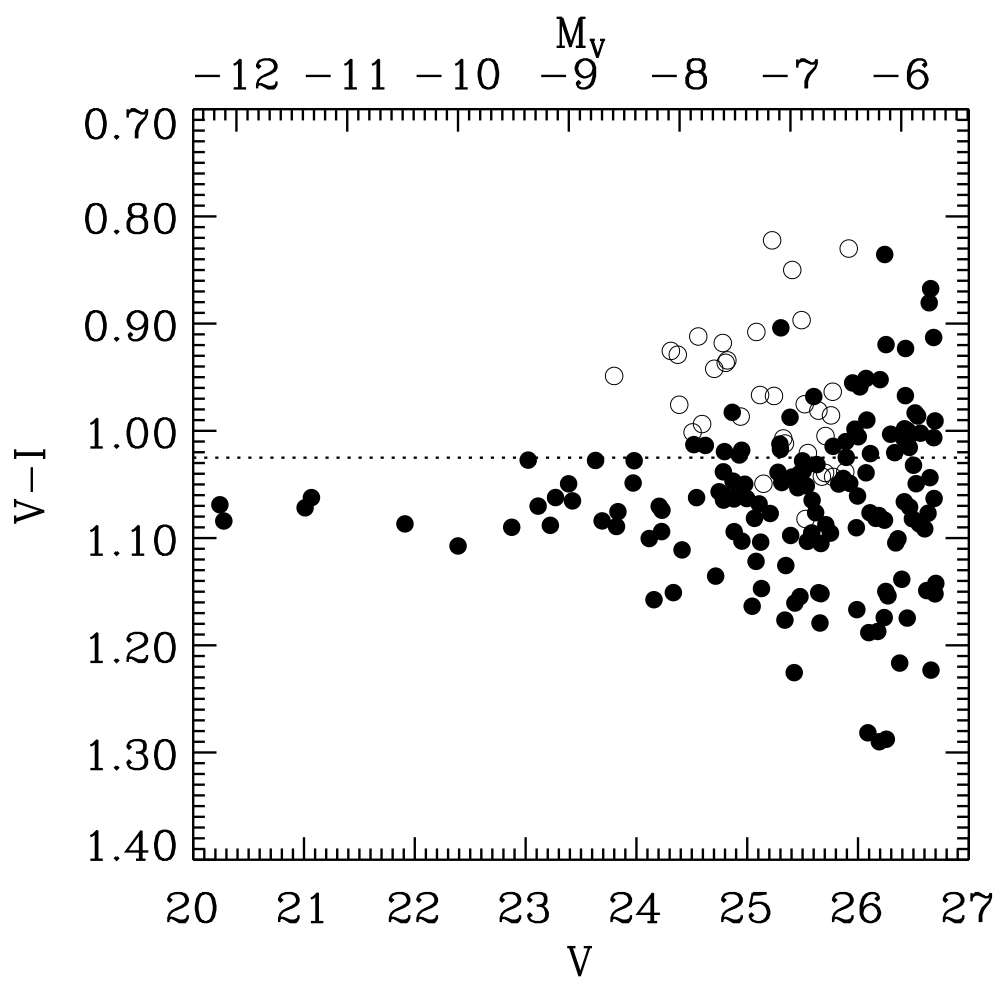

Fig. 12.- 


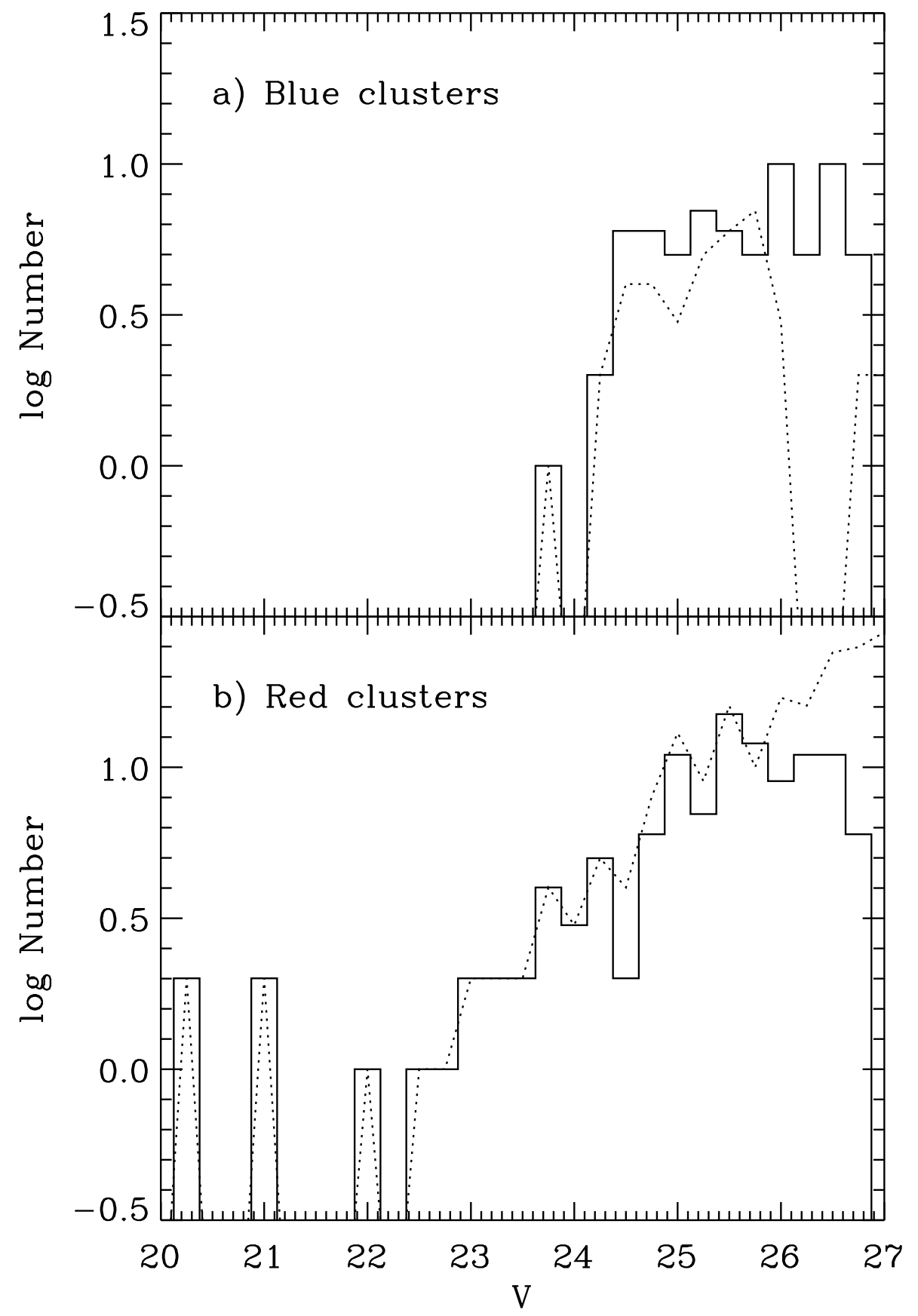

Fig. 13.- 
Table 1: Positions, magnitudes, and colors of the 50 brightest cluster candidates in NGC 3610

\begin{tabular}{|c|c|c|c|c|c|c|c|c|c|}
\hline$\#$ & Paper I $\#^{a}$ & $\begin{array}{r}\mathrm{RA} \\
(\mathrm{h}: \mathrm{m}: \mathrm{s})\end{array}$ & $\begin{array}{r}\text { DEC } \\
\left(\operatorname{deg}:^{\prime}:^{\prime \prime}\right)\end{array}$ & $\begin{array}{r}\text { Projected } \\
\text { Radius }{ }^{b} \\
\left(^{\prime \prime}\right)\end{array}$ & $\begin{array}{r}V^{c} \\
(\mathrm{mag})\end{array}$ & $\begin{array}{r} \pm^{d} \\
(\mathrm{mag})\end{array}$ & $\begin{array}{r}V-I^{c} \\
(\mathrm{mag})\end{array}$ & ${ }_{(\mathrm{mag})}^{ \pm^{d}}$ & $\begin{array}{l}\mathrm{M}_{V}{ }^{e} \\
(\mathrm{mag})\end{array}$ \\
\hline$(1)$ & $(2)$ & $(3)$ & (4) & (5) & $(6)$ & $(7)$ & $(8)$ & $(9)$ & (10) \\
\hline 1 & - & $11: 18: 25.27$ & $58: 47: 10.08$ & 1.14 & 20.78 & 0.12 & 1.30 & 0.17 & -11.61 \\
\hline 2 & 10 & $11: 18: 25.25$ & $58: 47: 12.35$ & 1.27 & 21.09 & 0.13 & 1.27 & 0.17 & -11.30 \\
\hline 3 & 1 & $11: 18: 27.96$ & $58: 47: 16.05$ & 21.95 & 21.53 & 0.05 & 1.05 & 0.05 & -10.86 \\
\hline 4 & - & $11: 18: 25.00$ & $58: 47: 12.82$ & 2.34 & 21.54 & 0.13 & 1.28 & 0.17 & -10.85 \\
\hline 5 & 2 & $11: 18: 24.57$ & $58: 47: 13.24$ & 5.40 & 21.70 & 0.06 & 1.14 & 0.06 & -10.69 \\
\hline 6 & - & $11: 18: 28.03$ & $58: 47: 05.11$ & 22.70 & 21.79 & 0.05 & 1.24 & 0.05 & -10.60 \\
\hline 7 & 8 & $11: 18: 27.46$ & $58: 47: 07.18$ & 17.91 & 21.92 & 0.05 & 1.10 & 0.05 & -10.47 \\
\hline 8 & - & $11: 18: 24.89$ & $58: 47: 13.59$ & 3.51 & 21.95 & 0.10 & 1.21 & 0.13 & -10.44 \\
\hline 9 & 3 & $11: 18: 33.84$ & $58: 46: 53.75$ & 69.31 & 21.96 & 0.05 & 1.15 & 0.05 & -10.43 \\
\hline 10 & 4 & $11: 18: 32.95$ & $58: 46: 15.41$ & 81.98 & 22.00 & 0.05 & 0.94 & 0.05 & -10.39 \\
\hline 11 & 7 & $11: 18: 23.79$ & $58: 47: 02.90$ & 13.74 & 22.09 & 0.05 & 1.19 & 0.05 & -10.30 \\
\hline 12 & - & $11: 18: 24.45$ & $58: 47: 26.87$ & 16.84 & 22.10 & 0.05 & 0.99 & 0.05 & -10.29 \\
\hline 13 & 6 & $11: 18: 25.70$ & $58: 47: 05.55$ & 6.73 & 22.11 & 0.06 & 1.16 & 0.06 & -10.28 \\
\hline 14 & - & $11: 18: 25.45$ & $58: 47: 11.09$ & 1.85 & 22.14 & 0.21 & 1.27 & 0.28 & -10.25 \\
\hline 15 & - & $11: 18: 25.41$ & $58: 47: 11.47$ & 1.62 & 22.14 & 0.22 & 1.14 & 0.32 & -10.25 \\
\hline 16 & - & $11: 18: 25.18$ & $58: 47: 13.01$ & 1.91 & 22.14 & 0.21 & 1.40 & 0.27 & -10.25 \\
\hline 17 & 5 & $11: 18: 27.34$ & $58: 46: 55.51$ & 22.73 & 22.39 & 0.05 & 1.19 & 0.05 & -10.00 \\
\hline 18 & 12 & $11: 18: 26.15$ & $58: 47: 14.31$ & 8.01 & 22.48 & 0.05 & 1.20 & 0.05 & -9.91 \\
\hline 19 & 9 & $11: 18: 25.88$ & $58: 47: 11.92$ & 5.30 & 22.48 & 0.06 & 1.15 & 0.06 & -9.91 \\
\hline 20 & 11 & $11: 18: 20.97$ & $58: 46: 33.72$ & 49.86 & 22.53 & 0.05 & 1.08 & 0.05 & -9.86 \\
\hline 21 & 23 & $11: 18: 25.55$ & $58: 47: 09.22$ & 3.28 & 22.57 & 0.27 & 1.14 & 0.40 & -9.82 \\
\hline 22 & 13 & $11: 18: 32.23$ & $58: 46: 15.43$ & 77.97 & 22.68 & 0.05 & 1.06 & 0.05 & -9.71 \\
\hline 23 & 14 & $11: 18: 25.17$ & $58: 47: 19.48$ & 8.37 & 22.68 & 0.05 & 1.08 & 0.05 & -9.71 \\
\hline 24 & 22 & $11: 18: 25.15$ & $58: 47: 14.11$ & 3.03 & 22.68 & 0.13 & 1.13 & 0.19 & -9.71 \\
\hline 25 & - & $11: 18: 19.13$ & $58: 46: 20.82$ & 69.03 & 22.71 & 0.05 & 1.03 & 0.05 & -9.68 \\
\hline 26 & 16 & $11: 18: 24.03$ & $58: 47: 14.80$ & 9.85 & 22.71 & 0.05 & 1.14 & 0.05 & -9.68 \\
\hline 27 & 29 & $11: 18: 25.64$ & $58: 47: 08.42$ & 4.27 & 22.72 & 0.16 & 0.97 & 0.26 & -9.67 \\
\hline 28 & 15 & $11: 18: 24.71$ & $58: 45: 53.33$ & 77.89 & 22.85 & 0.05 & 1.07 & 0.05 & -9.54 \\
\hline 29 & - & $11: 18: 25.34$ & $58: 47: 12.74$ & 1.92 & 22.89 & 0.27 & 1.26 & 0.38 & -9.50 \\
\hline 30 & 18 & $11: 18: 30.02$ & $58: 47: 28.42$ & 41.21 & 23.01 & 0.05 & 1.09 & 0.05 & -9.38 \\
\hline 31 & 17 & $11: 18: 26.45$ & $58: 46: 29.29$ & 42.92 & 23.08 & 0.05 & 1.18 & 0.05 & -9.31 \\
\hline 32 & 19 & $11: 18: 32.75$ & $58: 46: 03.85$ & 89.20 & 23.09 & 0.05 & 1.16 & 0.05 & -9.30 \\
\hline 33 & 20 & $11: 18: 25.69$ & $58: 46: 42.79$ & 28.57 & 23.31 & 0.05 & 1.26 & 0.05 & -9.08 \\
\hline 34 & 21 & $11: 18: 23.84$ & $58: 47: 23.44$ & 16.28 & 23.33 & 0.05 & 1.22 & 0.05 & -9.06 \\
\hline 35 & - & $11: 18: 25.52$ & $58: 46: 52.45$ & 18.82 & 23.34 & 0.05 & 1.17 & 0.06 & -9.05 \\
\hline 36 & - & $11: 18: 23.96$ & $58: 46: 52.65$ & 20.88 & 23.35 & 0.05 & 1.18 & 0.06 & -9.04 \\
\hline 37 & 25 & $11: 18: 25.21$ & $58: 47: 02.60$ & 8.51 & 23.36 & 0.06 & 1.13 & 0.06 & -9.03 \\
\hline 38 & 24 & $11: 18: 25.27$ & $58: 47: 02.34$ & 8.79 & 23.36 & 0.06 & 0.89 & 0.07 & -9.03 \\
\hline 39 & - & $11: 18: 24.88$ & $58: 47: 15.39$ & 4.99 & 23.43 & 0.17 & 1.02 & 0.25 & -8.96 \\
\hline 40 & 30 & $11: 18: 23.51$ & $58: 46: 11.92$ & 60.65 & 23.51 & 0.05 & 1.14 & 0.05 & -8.88 \\
\hline 41 & 28 & $11: 18: 26.28$ & $58: 45: 58.20$ & 73.39 & 23.55 & 0.05 & 0.91 & 0.05 & -8.84 \\
\hline 42 & 27 & $11: 18: 25.62$ & $58: 46: 39.96$ & 31.32 & 23.57 & 0.05 & 1.12 & 0.05 & -8.82 \\
\hline 43 & 36 & $11: 18: 23.62$ & $58: 47: 01.10$ & 15.90 & 23.61 & 0.05 & 1.16 & 0.05 & -8.78 \\
\hline 44 & 32 & $11: 18: 29.13$ & $58: 47: 09.59$ & 30.55 & 23.62 & 0.05 & 1.15 & 0.05 & -8.77 \\
\hline 45 & 37 & $11: 18: 25.83$ & $58: 46: 50.43$ & 21.24 & 23.65 & 0.06 & 1.13 & 0.06 & -8.74 \\
\hline 46 & 34 & $11: 18: 32.93$ & $58: 47: 38.61$ & 66.03 & 23.72 & 0.05 & 0.96 & 0.05 & -8.67 \\
\hline 47 & - & $11: 18: 24.54$ & $58: 47: 15.64$ & 6.90 & 23.73 & 0.13 & 1.38 & 0.17 & -8.66 \\
\hline 48 & - & $11: 18: 24.90$ & $58: 47: 09.79$ & 2.76 & 23.74 & 0.31 & 0.81 & 0.54 & -8.65 \\
\hline 49 & 33 & $11: 18: 31.40$ & $58: 46: 41.14$ & 56.71 & 23.78 & 0.05 & 1.34 & 0.05 & -8.61 \\
\hline 50 & - & $11: 18: 31.40$ & $58: 45: 48.25$ & 95.83 & 23.79 & 0.05 & 0.63 & 0.06 & -8.60 \\
\hline
\end{tabular}

${ }^{a}$ Corresponding ID number from Table 4 of Paper I. Note how the new search algorithm is able to identify new clusters close to the center of NGC 3610. Most of the missing clusters in the outer regions are due to different orientations of the two sets of observations.

${ }^{b}$ Using a central position of $11 \mathrm{~h} 18 \mathrm{~m} 25.209 \mathrm{~s}$ and $58^{\mathrm{deg}} 47^{\prime} 11.12^{\prime \prime}$.

${ }^{c}$ No correction for extinction was made since Burstein and Heiles (1984) give $\mathrm{A}_{V}=0.00$ mag.

${ }^{d}$ A value of $0.05 \mathrm{mag}$ has been added in quadrature to approximate the error introduced by uncertainties in zeropoint, aperture corrections, and CTE corrections.

${ }^{e}$ Using $\mathrm{H}_{0}=75 \mathrm{~km} \mathrm{~s}^{-1} \mathrm{Mpc}^{-1}$ and $\mathrm{m}-\mathrm{M}=32.39$. 